\title{
Infectious alphavirus production from a simple plasmid transfection
}

\author{
J Jordan Steel ${ }^{1}$, Brittney R Henderson', Siddhi BC Lama', Ken E Olson ${ }^{1}$ and Brian J Geiss ${ }^{1,2^{*}}$
}

\begin{abstract}
We have developed a new method for producing infectious double subgenomic alphaviruses from plasmids transfected into mammalian cells. A double subgenomic Sindbis virus (TE3'2J) was transcribed from a cytomegalovirus Polll promoter, which results in the production of infectious virus. Transfection of as little as 125 ng of plasmid is able to produce $1 \times 10^{8}$ plaque forming units $/ \mathrm{ml}(\mathrm{PFU} / \mathrm{ml})$ of infectious virus 48 hours posttransfection. This system represents a more efficient method for producing recombinant Sindbis viruses.
\end{abstract}

Keywords: Sindbis, Alphavirus, Virus production, Gateway

\section{Background}

Alphaviruses (Family: Togaviridae) are used extensively in molecular biology as tools for gene expression and delivery [1]. Alphaviruses can infect a wide range of species and have small manipulable genomes that can encode and express heterologous genes [2,3]. Alphaviruses possess a positive sense capped RNA that is approximately $11.6 \mathrm{~kb}$ in length. The 5' end of the viral RNA is translated into 4 nonstructural proteins (nsP 14) which are involved in replicating the viral genome. A negative strand RNA is replicated from the full-length positive strand viral RNA that contains a subgenomic promoter (SGP) that drives transcription of the $26 \mathrm{~S}$ subgenomic RNA. The subgenomic RNA encodes the viral structural proteins (Capsid, E3, E2, 6K, and E1) necessary for virion assembly [4]. The SGP has previously been duplicated in the viral genome, allowing for heterologous genes to be expressed from the virus is the same fashion as the viral structural proteins [5]. Heterologous genes that have been engineered into alphavirus genomes include fluorescent proteins, luciferases, cellular proteins, antisense RNAs, and ribozymes [6-12]. Engineering a heterologous gene or RNA behind the second subgenomic promoter allows for the production of a fully infectious virus simultaneous with the expression of the heterologous gene in a wide range of species.

\footnotetext{
* Correspondence: brian.geiss@colostate.edu

'Department of Microbiology, Immunology, and Pathology, 1682 Campus

Delivery, Colorado State University, Fort Collins, CO 80523 USA

Full list of author information is available at the end of the article
}

The current method used to create a recombinant double-subgenomic virus that expresses a heterologous gene is somewhat inefficient. To insert the gene of interest (GOI) into the virus, the viral infectious clone plasmid is digested with a unique restriction enzyme and the PCR amplified GOI is restriction enzyme digested and ligated into the virus infectious clone plasmid. This approach usually results in the GOI ligating in either the sense or antisense orientation, requiring screening of the resulting clones for the orientation of the insert. Of additional concern with single-site restriction cloning is multiple copies of the GOI ligating into the virus infectious clone plasmid if small inserts are used. Once a clone with the GOI in the correct orientation has been identified and sequenced, the plasmid is linearized using a unique restriction site at the end of the viral genome to allow for run-off RNA transcription. Several micrograms of phenol-chloroform extracted plasmid DNA is used in in vitro RNA transcription reactions with a nucleotide cap analog to generate capped viral RNAs. The RNA is then either electroporated into cells or transfected with chemical or liposomal RNA transfection reagents, and virus is collected from culture media 2472 hours later.

Several points in this process reduce efficiency and increase time of virus production. Insertion of a GOI into the viral genome by restriction cloning is relatively inefficient due to the need to screen for insert orientation. In vitro RNA transcription kits that are commonly used are expensive and generally result in low yields of
C Biomed Central 
full length capped RNAs (B. Geiss, personal observation). Additionally, phage DNA-dependent RNA polymerases (such as T7 and SP6) have low fidelity and can result in quasi-species from the in vitro transcription reaction [13]. Electroporation of cells with RNA requires large numbers of cells $\left(1-5 \times 10^{6}\right.$ cells/electroporation), is sensitive to salt concentration that can damage cells during electroporation, and require specialized equipment not always available in laboratories. Chemical and liposomal RNA transfection has been used more recently to avoid using electroporation, but RNA degradation during transfection is still a concern.

To make alphavirus expression systems easier to use and more accessible to researchers, we have developed virus expression plasmids that are simple to manipulate and can rapidly and inexpensively produce infectious virus. Building on our previous work with Sindbis virus replicon expression plasmids [14], we generated a double-subgenomic Sindbis virus expression plasmid that transcribes RNA from a cytomegalovirus (CMV) PolII promoter and cleaves the RNA at the 3' end of the viral genome similar to plasmid-based replicon expression systems [14-16]. In addition, we have developed variants of this system that utilize recombination technology to rapidly and efficiently insert a GOI into the virus in the desired orientation. The negative and positive selection capability of the Gateway cloning system makes it attractive for rapid GOI cloning. Using this system we have produced several reporter gene expressing viruses and demonstrate their use in cell culture.

\section{Methods \\ Plasmid Construction}

The base TE/3'2J Sindbis virus expression plasmid (pBG167) was constructed by digesting a TE/3'2J replicon expression plasmid pBG68 [14] with HpaI and XbaI restriction enzymes and ligating the vector with T4 DNA ligase to a $4631 \mathrm{bp} \mathrm{XbaI/HpaI} \mathrm{fragment} \mathrm{from} \mathrm{the} \mathrm{pTE/3'2J}$ infectious clone [17]. pBG218 was created by ligating NheI flanked GFP open reading frame into the unique XbaI site in pBG167. The orientation of the GFP insert was verified by sequencing with BG626 (5' CACCTCTAGACCATGGATCC) and BG583 (5' CTAGATAAATGGTTAATATAGT). pBG167-based recombination ready plasmids were generated by ligating a PCR amplified attR1/attR2 recombination cassette from Gateway pDEST32 (Invitrogen) into pBG167. BG121 (5' CATGGCTAGCACAA GTTTGTACAAAAAAGCTGAACG) and BG122 (5' CATGGCTAGCACCACTTTGTACAAGAAAGCTGA ACG) contain NheI restriction sites, and were used to ligate the recombination cassette into $\mathrm{XbaI}$ digested pBG167 and were transformed into ccdB resistant DB 3.1 E. coli cells (Invitrogen). Forward and reverse attR1/attR2 recombination cassettes were identified by DNA sequencing and resulted in pBG210 and pBG211, respectively. pBG440 (pENTR-D/Topo-GFP) and pBG403 (pENTR-D/Topo-Renilla Luciferase) were constructed by PCR amplifying the eGFP gene from pIE-GFP (Clontech) with primers BG518 (5' CACCGCTAGCATGGGGATGCATGGTACCATGG) and BG519 (5' AAGTGCTAGCTTACTTGTACAGCTCGTCCATGCC) or the Renilla luciferase gene from $\mathrm{pWN} 5$ 'RucPur [18] with primers BG556 (5' CACCATGGCTAGCAAGGTGTACGACC) and BG557 (5' CTACTGCTCGTTCTTCAGCACG) and incubating the gel extracted PCR products with pENTRD/Topo. pBG344 (pENTR-D/TOPO-mCherry) was produced by incubating the mCherry gene PCR amplified from pmCherry (Clontech) with primers BG504 (5' CACCAGATCTATGGTGAGCAAGGGCGAGGAGG) and BG505 (5' CATGAGATCTTTACCGGTGCTTGTACAGCTCGTCC) with pENTR-D/Topo. BG212 was generated by performing a LR Clonase II reaction between pBG440 and pBG210, pBG451 was generated from a LR Clonase II Reaction between pBG403 and pBG210, and pBG452 was generated from a LR Clonase II reaction between pBG344 and pBG210.

All viral sequences in pBG167 plasmid were verified with a panel of Sindbis-specific primers. Inserts ligated into the unique XbaI site in pBG167 were sequenced with primers BG 583 (5' CTAGATAAATGGTTAATATAGT) and BG626 (5' CACCTCTAGACCATGGATCC) to verify sequence and orientation. PCR products that were Topo-cloned into pENTR-D/Topo were sequenced with M13 ${ }_{-20}$ and M13 Reverse primers. All Gateway attR1/attR2 containing plasmids were grown on $\operatorname{ccdB}$ resistant DB3.1 E. coli cells (Invitrogen), and all other plasmids were grown in DH5 $\alpha$ E. coli cells. Diagrams of each virus expression construct are provided in Figure 1.

\section{Cell Culture and Transfection}

Baby Hamster Kidney (BHK) and Vero cells were maintained in Hyclone DMEM supplemented with $10 \%$ fetal bovine serum (FBS), 5\% Pen/Strep, and 5\% L-Glutamine [18]. These mammalian cells were kept in a $37^{\circ} \mathrm{C}$ incubator with $5 \% \mathrm{CO}_{2}$. BHK cells were plated in 6-well plates for transfection with Lipofectamine 2000 (Invitrogen). Cells were transfected at $60 \%$ confluency with 125 ng DNA per well following the manufacturer's recommendations. Transfection media was removed and replaced with fresh media 6-8 hours post transfection. Aedes albopictus C6/36 cells were cultured in L-15 medium with $10 \% \mathrm{FBS}, 5 \%$ Pen/Strep, and 5\% L-Glutamine and were maintained at $28^{\circ} \mathrm{C}$ [19].

\section{Plaque Assays and Growth Curves}

Viral titers were determined using plaque assay titrations on BHK cells as described previously [20]. BHK cells seeded on 24 well tissue culture plates were 


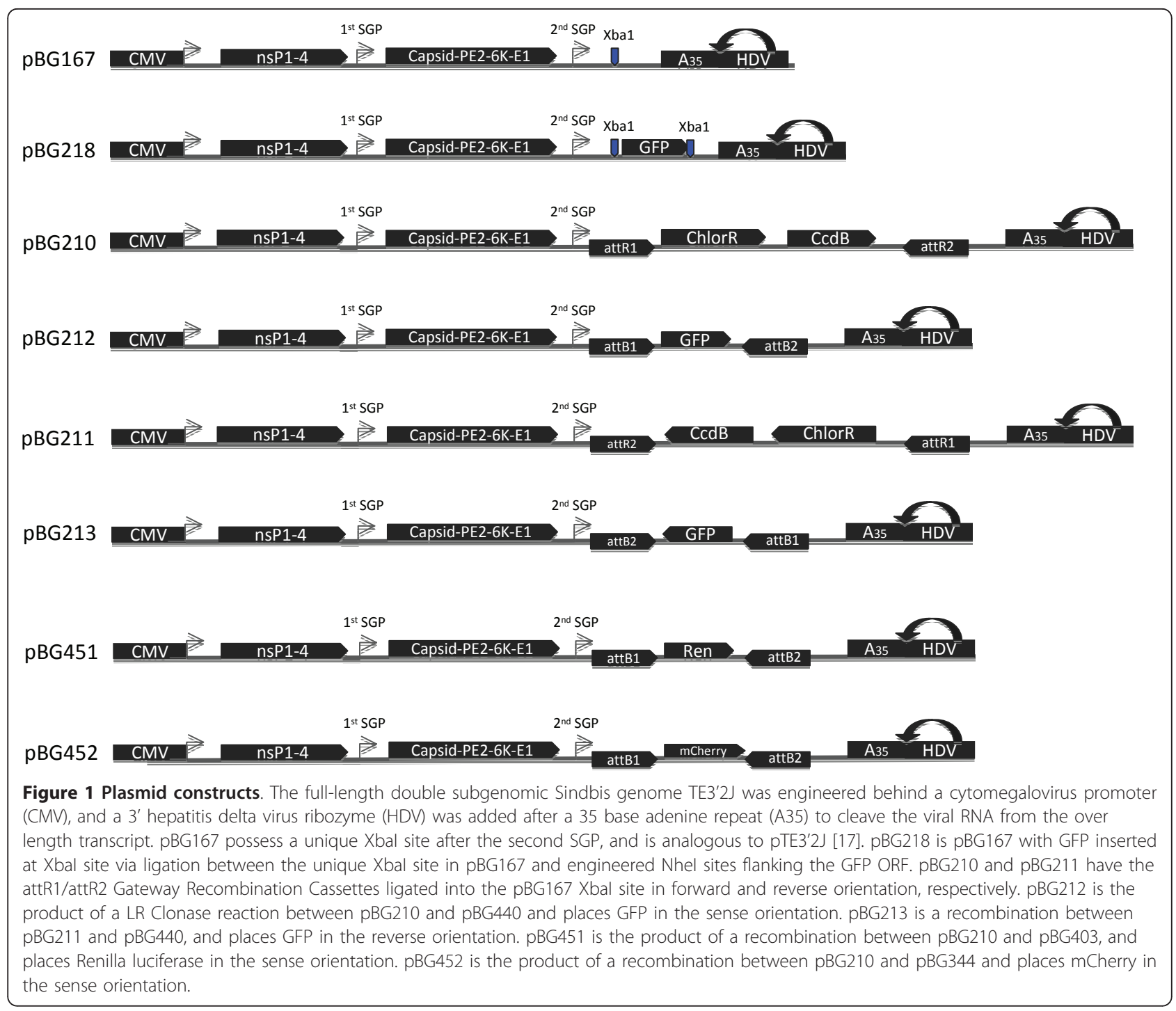

infected with serial dilutions of virus samples for 1 hour at $37^{\circ} \mathrm{C}$, and then an agarose nutrient overlay was added. Cells were maintained at $37^{\circ} \mathrm{C}$ for 3 days for visible plaques to develop. On day 3, Thiazolyl Blue Tetrazolium Blue (MTT) at $5 \mathrm{mg} / \mathrm{ml}$ in PBS was added to the overlay to visualize plaques and incubated at $37^{\circ} \mathrm{C}$ for $12 \mathrm{hrs}$. Viral plaques were counted and titers determined as plaque forming units $(\mathrm{PFU}) / \mathrm{ml}$.

P0 (transfection initiated) and P1 (virus initiated) growth curves were performed in 6-well plates. For P0 growth curves, BHK cells were transfected as described above. The cells were washed with media to remove excess transfection complexes, and $500 \mu \mathrm{l}$ samples were collected at $4,8,12,24,36,48,60$, and 72 hours post transfection. Sample volumes collected were replaced with fresh media to maintain a total volume of $2 \mathrm{ml}$. Aliquots of samples were stored at $-80^{\circ} \mathrm{C}$ until titration or infection. For P1 growth curves, titered P0 derived virus ( $48 \mathrm{hr}$ post-transfection) was added to BHK or Vero cells at MOI $=0.1$ or 0.01 as indicated. Samples were collected and analyzed as described for P0 growth curves. Each growth curve was replicated three times, and average titers and standard error calculated. Data was graphed using Microsoft Excel.

\section{GFP, mCherry, and Renilla Luciferase expression}

GFP and mCherry expressing cells were imaged on an inverted Nikon Photopt fluorescence microscope with a CoolSnap CCD camera using either $488 \mathrm{nM} / 535 \mathrm{nM}$ filters for GFP detection, $560 \mathrm{nM} / 630 \mathrm{nM}$ filters for mCherry detection, or phase contrast for cell imaging. Image contrast was adjusted for all images equally using ImageJ software. Luciferase assays were performed using Viviren Live Cell Renilla Luciferase Reagent (Promega) 
in white opaque 96-well plates. Briefly, at the indicated times media was removed from the infected wells and replaced with $25 \mu \mathrm{L}$ DMEM supplemented with $37 \mu \mathrm{g} /$ ul Viviren reagent. The plates were incubated at $37^{\circ} \mathrm{C}$ for 10 minutes, then relative light units (RLU) in each well were determined on a Victor $3 \mathrm{~V}$ Multimode platereader (Perkin Elmer). All experiments were performed at least 3 independent times, and averages and standard errors are reported.

\section{Results and Discussion}

\section{Production of infectious Sindbis viruses from plasmid transfections}

To test if infectious Sindbis virus could be produced from plasmid pBG167 (Figure 1), 125 ng of pBG167 plasmid was transfected with Lipofectamine 2000 into a single well of BHK cells in a 6 -well plate. 4 hrs post transfection the cells were washed with media to remove excess transfection complexes, and media samples were collected at the indicated times post transfection (Figure $2 A)$. Viral growth kinetics and maximal viral titers are similar to TE3'2J produced from RNA transcripts [9,21], indicating that a simple transfection of Sindbis virus expression plasmid produces similar amounts of virus as traditional production methods.

Once we determined that infectious Sindbis virus could be produced directly from transfected pBG167 plasmid, we generated a series of constructs with GFP inserted behind the 3' SGP either by ligation into the XbaI site (pBG218) or by recombination into a Gateway attR1/ attR2 recombination cassette in plasmids pBG210 or pBG211 (pBG212, and pBG213). These plasmids were stably propagated in $E$. coli in the pcDNA3.1 vector backbone with average plasmid miniprep yields of $100 \mathrm{ng} / \mu \mathrm{l}$ (data not shown), and were able to produce infectious virus in passage 0 (P0) growth curves (Figure $2 \mathrm{~A}$ ). We observed that the maximal titers for each GOI containing clone was lower than pBG167-derived virus, which has been reported previously from transcription-derived TE3'2J viruses [9]. To determine if the plasmid derived viruses were able to replicate in a second passage (P1), we infected BHK cells with each P0 virus at $\mathrm{MOI}=0.01$ and determined growth kinetics (Figure 2B). The P1 viral growth kinetics and maximal titers were much more uniform that the P0, which may be accounted for by differing molar amounts of plasmid being transfected in the P0 samples due to differing plasmids sizes. Therefore the system was able to produce infectious virus.

\section{Reporter Gene Expression}

We next tested reporter gene expression from each virus. pBG212 and pBG218 derived virus both produced GFP expression as visualized by fluorescence microscopy, whereas pBG213 derived virus with GFP inserted in reverse orientation and pBG167 did not express GFP (Figure 3A). We also tested the kinetics of GOI expression using P0 Renilla luciferase expressing virus derived from pBG451 on BHK cells. The kinetics of Renilla luciferase expression in the P1 infection closely followed the kinetics of virus production in growth curve assays (Figure $3 \mathrm{~B}$ ), indicating that viral replication and reporter gene expression were closely linked. The slight delay in PFU production as compared to Renilla luciferase activity at $8 \mathrm{hrs}$ post infection likely reflects that the subgenomic promoters are functioning and producing Renilla luciferase and viral structural proteins, but the virions had not had time to assemble into infectious viruses at that point. To verify that reporter gene expression is sensitive to a known viral replication inhibitor, we treated cells with the cellular calmodulin kinase inhibitor W-7 (N-(6-Aminohexyl)-5-chloro-1-naphthalenesulfonamide hydrochloride) that had previously been shown to
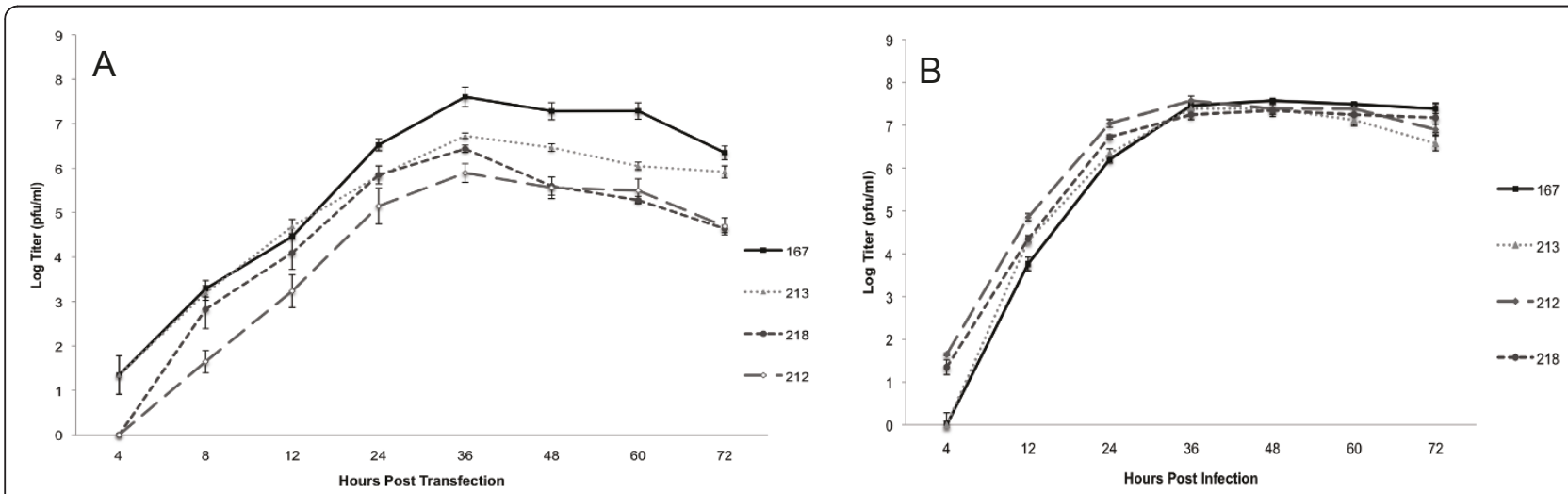

Figure 2 Production of Infectious Sindbis Viruses. A) P0 viral titers from plasmid transfected BHK cells. 125 ng of plasmid DNA transfected with Lipofectamine 2000 reagents. Media samples were collected at the indicated times and viral titers were determined by plaque assays as plaque forming units (PFU)/ml. B) P1 viral titers in BHK cells. BHK cells were infected at MOI $=0.01$ with 48 hr PO viruses, then samples were collected at the indicated times and viral titers were determined by plaque assay. 


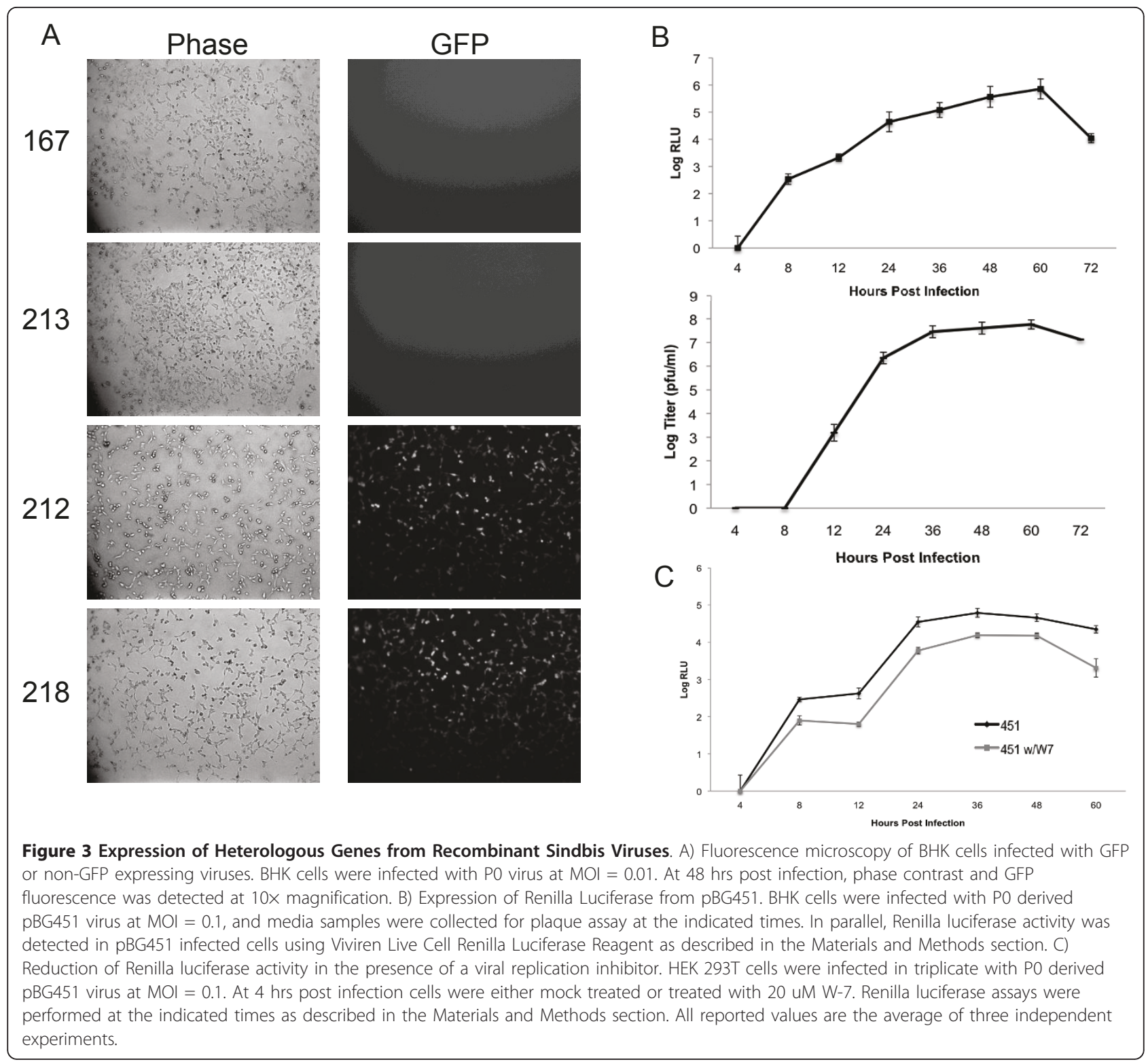

interfere with viral packaging [22]. $20 \mu \mathrm{M} \mathrm{W}-7$ treatment reduced reporter gene expression by about $1 \mathrm{log}$ in P1 infections as compared to untreated samples (Figure $3 \mathrm{C}$ ), demonstrating that Renilla luciferase expression was linked to viral replication.

\section{Replication of plasmid derived virus in various cell lines}

Sindbis is known for broad tropism and is able to infect different species with relatively similar efficiencies. We determined if plasmid-derived virus was able to replicate well in cell lines other than BHK cells. Renilla luciferase expressing pBG451 virus (P0) was used to infect BHK (murine), Vero (primate), and C6/36 (mosquito) cell lines at MOI $=0.1$, and viral titers and Renilla luciferase activity were assessed at $48 \mathrm{hrs}$ post infection (P1)
(Figure 4). BHK cells and Vero cells produced similar titers $48 \mathrm{hrs}$ post infection $\left(\sim 1 \times 10^{6} \mathrm{PFU} / \mathrm{ml}\right)$, and showed similar Renilla luciferase signals at $48 \mathrm{hrs}(\sim 1 \times$ $\left.10^{5} \mathrm{RLU}\right)$. C6/36 cells produced lower titers and Renilla luciferase signal than BHK and Vero cells at 48 hrs post infection, though this difference may be due to slower growth kinetics in invertebrate cell lines. These data indicate that the P0 virus was able to effectively infect and replicate in both murine, primate, and mosquito cell lines, and that the Renilla luciferase reporter gene was expressed in each cell line during the P1 infection.

\section{Stability of Reporter Gene Expression}

To test the stability of the att-containing viruses, we passaged the mCherry containing pBG452 virus 3 


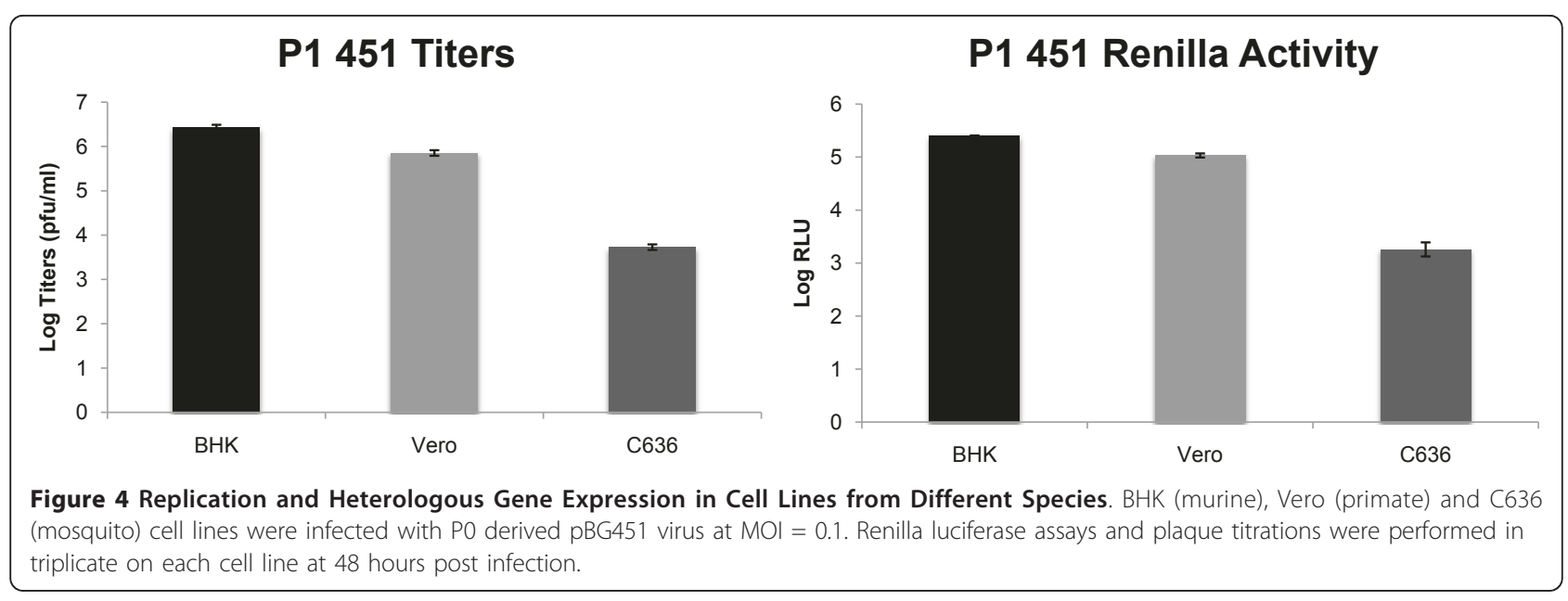

times in either BHK or $\mathrm{C} 6 / 36$ cells using $\mathrm{MOI}=0.01$ for each infection. We collected media after 48 hrs for each passage, and after the $3^{\text {rd }}$ passage we infected naïve BHK or C6/36 cells with virus from passage at MOI $=0.01$. We observed that each cell type showed equal fluorescence with each passage at $28 \mathrm{hrs}$ postinfection (BHK) and 86 hrs post-infection (C6/36) (Figure 5), indicating that the att-flanked GOI remained stable over the course of at least 3 passages in both $\mathrm{BHK}$ and $\mathrm{C} 6 / 36$ cells.

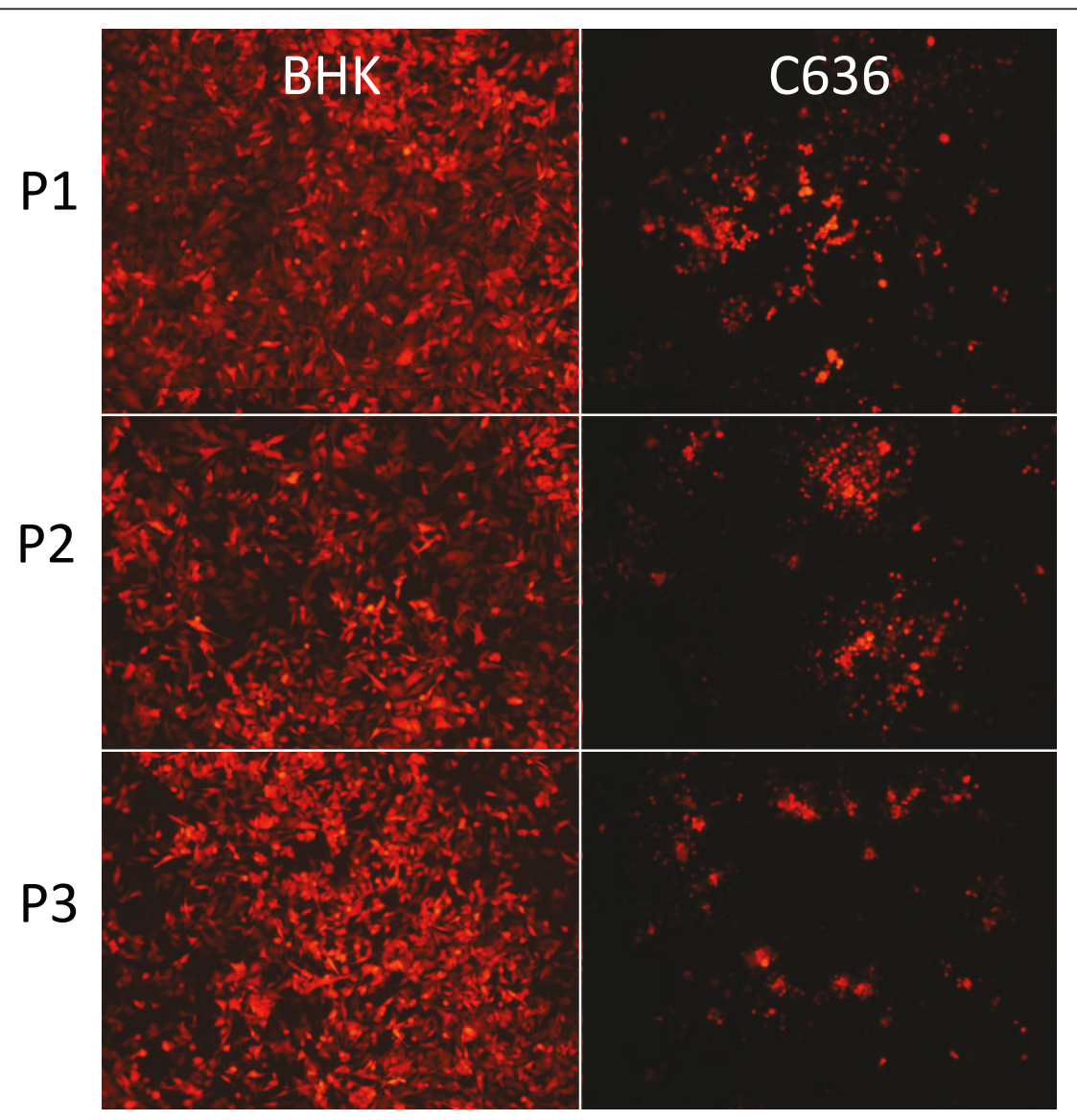

Figure 5 Stability of Reporter Gene Expression. BHK or C636 cell lines were infected with P0 derived pBG452 virus at MOI = 0.01 for 48 hrs, then media was collected and titered. This process was repeated for a total of 3 passages. After the $3^{\text {rd }}$ passage, BHK or C6/36-derived virus from each passage was added to BHK or C6/36 cells at $\mathrm{MOI}=0.01$ and incubated for 28 (BHK) or 86 (C6/36) hrs. mCherry fluorescence was detected at the indicated times by fluorescence microscopy. 
The typical sequence of events for production of a GOI expressing TE'32J virus takes approximately 3-4 weeks. Cloning and inserting the GOI 3' of the viral subgenomic promoter usually takes $\sim 2$ weeks from the PCR amplification step and requires screening clones for insert orientation. Once a clone has been identified and the GOI sequenced, the production of infectious RNA takes 2-3 additional days due to preparation of linearized maxiprepped plasmid DNA for in vitro transcription and electroporation of RNA transcripts into $\sim 2-10 \times 10^{6}$ cells using expensive electroporation systems. Additionally, because RNA is being used, extra precautions to reduce RNase degradation of transcripts must be used. Therefore, the traditional production of GOI expressing TE3'2J can be time consuming and somewhat expensive.

Our system circumvents many of the problems associated with production of GOI expressing TE3'2J viruses. GOI PCR products can be rapidly cloned into attL containing pENTR-D/Topo vectors, then the GOI can be recombined into pBG210 (forward) or pBG211 (reverse) TE3'2J expression plasmids with high efficiency. In our hands this process take about 1 week and is highly efficient. The pENTR-D/Topo vector and LR Clonase II systems from Invitrogen are relatively expensive, but each reaction can be scaled down to reduce costs and extend the number of reactions that can be performed. We have successfully recombined short hammerhead ribozymes (67 bp) and the large Firefly luciferase gene $(1.6 \mathrm{~Kb}$ ) into pBG210 (data not shown), indicating that a wide range of insert sizes can be accommodated. Once the GOI containing virus expression plasmid has been constructed, virus is produced by transfecting a small amount of the plasmid into BHK cells using common transfection agents and $2 \times 10^{5}$ cells in a single well. Virus is produced within a few days with minimal effort. The format for transfection can be adjusted from 96-well plates to $T_{150}$ flasks as needed, making virus production very flexible and rapid. GOI expression was verified from several viruses, including viruses that produce GFP and Renilla luciferase (Figures 3 and 4). The kinetics of Renilla luciferase expression closely mirrored viral replication kinetics, indicating that the GOI is stable and can be used as a readout for viral replication as previously described [7-9].

\section{Conclusions}

In this report we describe the construction and characterization of a new Sindbis (TE3'2J) virus production system. The virus expression plasmids we describe have several features that make them useful for rapidly generating Sindbis viruses that express genes of interest. We have developed a system by which fully infectious reporter gene expressing virus can be produced simply by transfecting a small amount of virus expression plasmid into cultured mammalian cells. Genes of interest can be rapidly incorporated into viruses in specific orientations via Gateway recombination. High-titer virus produced from our system can infect multiple cell types in culture and maintain reporter gene expression. The ease of cloning and specificity of insert orientation would make this system ideal for generating libraries of infectious viruses expressing randomized trans-cleaving ribozymes or inverted cDNA libraries to screen for host genes that are involved in viral replication or antiviral responses. In addition, the ability to launch virus production directly in cells with stable plasmid DNA may open up the possibility of using these constructs for stable Sindbis virus vaccines that can be launched via plasmid injection that may provide more robust immune responses than non-spreading replicon vaccines.

\section{Acknowledgements}

We would like to thank members of the Arthropod-Borne and Infectious Diseases Laboratory (AIDL) for helpful discussions. In particular we thank Aaron Phillips for technical assistance. This work was funded by grant Al04643 from the National Institutes of Health (NIAID) to KEO and BJG.

\section{Author details}

'Department of Microbiology, Immunology, and Pathology, 1682 Campus Delivery, Colorado State University, Fort Collins, CO 80523 USA. ${ }^{2}$ Department of Biochemistry and Molecular Biology, 1870 Campus Delivery, Colorado State University, Fort Collins, CO 80523 USA.

\section{Authors' contributions}

JS constructed and tested the virus expression plasmids, helped design the study, and helped write the manuscript. BRH and SBCL helped perform plaque and luciferase assays during. KEO and BJG provided funding for the manuscript, and BJG helped write the manuscript and design the overall project. All authors have read and approved this manuscript.

\section{Competing interests}

The authors declare that they have no competing interests.

Received: 25 March 2011 Accepted: 19 July 2011

Published: 19 July 2011

\section{References}

1. Lundstrom K: Alphavirus in Gene Therapy. Viruses 2009, 1:13-25.

2. Huang HV, Rice CM, Xiong C, Schlesinger S: RNA viruses as gene expression vectors. Virus Genes 1989, 3:85-91.

3. Xiong C, Levis R, Shen P, Schlesinger S, Rice CM, Huang HV: Sindbis virus: an efficient, broad host range vector for gene expression in animal cells. Science 1989, 243:1188-1191.

4. Strauss JH, Strauss EG: The alphaviruses: gene expression, replication, and evolution. Microbiol Rev 1994, 58:491-562.

5. Frolov I, Frolova E, Schlesinger S: Sindbis virus replicons and Sindbis virus: assembly of chimeras and of particles deficient in virus RNA. J Virol 1997, 71:2819-2829.

6. Cirimotich CM, Scott JC, Phillips AT, Geiss BJ, Olson KE: Suppression of RNA interference increases alphavirus replication and virus-associated mortality in Aedes aegypti mosquitoes. BMC Microbiol 2009, 9:49.

7. Cook SH, Griffin DE: Luciferase imaging of a neurotropic viral infection in intact animals. J Virol 2003, 77:5333-5338.

8. Olson KE, Higgs S, Hahn CS, Rice CM, Carlson JO, Beaty BJ: The expression of chloramphenicol acetyltransferase in Aedes albopictus $(\mathrm{C} 6 / 36)$ cells 
and Aedes triseriatus mosquitoes using a double subgenomic recombinant Sindbis virus. Insect Biochem Mol Biol 1994, 24:39-48.

9. Pierro DJ, Myles KM, Foy BD, Beaty BJ, Olson KE: Development of an orally infectious Sindbis virus transducing system that efficiently disseminates and expresses green fluorescent protein in Aedes aegypti. Insect Mol Biol 2003, 12:107-116.

10. Smith $S M$, Maldarelli F, Jeang KT: Efficient expression by an alphavirus replicon of a functional ribozyme targeted to human immunodeficiency virus type 1. J Virol 1997, 71:9713-9721.

11. Uhlirova M, Foy BD, Beaty BJ, Olson KE, Riddiford LM, Jindra M: Use of Sindbis virus-mediated RNA interference to demonstrate a conserved role of Broad-Complex in insect metamorphosis. Proc Natl Acad Sci USA 2003, 100:15607-15612.

12. Travanty EA, Adelman ZN, Franz AW, Keene KM, Beaty BJ, Blair CD, James AA, Olson KE: Using RNA interference to develop dengue virus resistance in genetically modified Aedes aegypti. Insect Biochem Mol Biol 2004, 34:607-613.

13. Pugachev KV, Guirakhoo F, Ocran SW, Mitchell F, Parsons M, Penal C, Girakhoo S, Pougatcheva SO, Arroyo J, Trent DW, Monath TP: High fidelity of yellow fever virus RNA polymerase. J Virol 2004, 78:1032-1038.

14. Geiss BJ, Shimonkevitz LH, Sackal Cl, Olson KE: Recombination-ready Sindbis replicon expression vectors for transgene expression. Virol J 2007, 4:112.

15. Ivanova L, Schlesinger S, Olivo PD: Regulated expression of a Sindbis virus replicon by herpesvirus promoters. J Virol 1999, 73:1998-2005.

16. Dubensky TW Jr, Driver DA, Polo JM, Belli BA, Latham EM, Ibanez CE, Chada S, Brumm D, Banks TA, Mento SJ, et al: Sindbis virus DNA-based expression vectors: utility for in vitro and in vivo gene transfer. $J$ Virol 1996, 70:508-519.

17. Hahn CS, Hahn YS, Braciale TJ, Rice CM: Infectious Sindbis virus transient expression vectors for studying antigen processing and presentation. Proc Natl Acad Sci USA 1992, 89:2679-2683.

18. Geiss BJ, Pierson TC, Diamond MS: Actively replicating West Nile virus is resistant to cytoplasmic delivery of siRNA. Virol J 2005, 2:53.

19. Adelman ZN, Sanchez-Vargas I, Travanty EA, Carlson JO, Beaty BJ, Blair CD, Olson KE: RNA silencing of dengue virus type 2 replication in transformed C6/36 mosquito cells transcribing an inverted-repeat RNA derived from the virus genome. J Virol 2002, 76:12925-12933.

20. Hernandez R, Sinodis C, Brown DT: Sindbis virus: propagation, quantification, and storage. Curr Protoc Microbiol 2005, Chapter 15, Unit 15B 11.

21. Heise MT, White $L$, Simpson DA, Leonard C, Bernard KA, Meeker RB, Johnston RE: An attenuating mutation in nsP1 of the Sindbis-group virus S.A.AR86 accelerates nonstructural protein processing and up-regulates viral 26S RNA synthesis. J Virol 2003, 77:1149-1156.

22. Liu N, Brown DT: Phosphorylation and dephosphorylation events play critical roles in Sindbis virus maturation. Virology 1993, 196:703-711.

doi:10.1186/1743-422X-8-356

Cite this article as: Steel et al: Infectious alphavirus production from a simple plasmid transfection. Virology Journal 2011 8:356.

\section{Submit your next manuscript to BioMed Central and take full advantage of:}

- Convenient online submission

- Thorough peer review

- No space constraints or color figure charges

- Immediate publication on acceptance

- Inclusion in PubMed, CAS, Scopus and Google Scholar

- Research which is freely available for redistribution

Submit your manuscript at www.biomedcentral.com/submit
Biomed Central 